\title{
Intervention Strategy Applied ICF Checklists for Sitting Cross- Legged in Patient with Multiple Ligament Knee Injury: Single Subject Study
}

\author{
Chan Yang Kim¹, Jung-Won Kwon²
}

'Department of Exercise Prescription Rehabilitation, Dankook University, Cheonan, Republic of Korea; ${ }^{2}$ Department of Physical Therapy, College of Health Sciences, Dankook University, Cheonan, Republic of Korea

Purpose: This study applies the ICF to identify the patient's body function, structure, and participation, evaluates the patient's environmental factors and individual factors, and is a high level of movement to return to the society of patients with multiple ligament injury of the knee joint.

Methods: Progressive strength training and ROM exercise were performed 30 minutes a day, 5 times a week for 6 weeks. The evaluation was performed by examining the ROM, length, MMT, instability, dynamic balance, pain and depression.

Results: The ROM of the knee joint was improved from $110^{\circ}$ to $135^{\circ}$ after intervention, and the knee flexion length decreased from $69 \mathrm{~cm}$ to $45 \mathrm{~cm}$. Knee flexor is Good after intervention from Poor-, and knee extensor is Good+ after intervention from Poor, and the plantar flexor of the ankle joint improved from Poor- before intervention to Good after intervention and dorsi-flexor of the ankle joint improve to Good from Poor. Pain index was moderate before and after the intervention, with a score of 3, 2 after the intervention, and when maintaining the sitting cross-legged, the before intervention score was 7 to 4 after the intervention.

Conclusion: The patient's posture of sitting cross-legged was maintained from 30 seconds before intervention to 14 minutes after intervention. These results were able to set the hypothesis design, intervention method and goal that the multifaceted approach of environment and individual factors as well as body function and structure area, activity and participation area using ICF checklists, it is helped the patient to return to daily life.

Keywords: ICF, Sitting cross-legged, Multiple ligament injury

\section{INTRODUCTION}

Therapists need to collect a lot of information from patient for effective intervention to evaluate and analyze the problem, as well as to make accurate decisions to solve the problems.

International Classification of Functioning (ICF) is divided into body functions and structure areas that represent changes in the body's physiological functions and anatomical structures, and activities and participation areas that include the ability to perform tasks. ${ }^{1}$ In addition, because it is divided into environmental factors and individual factors, it is possible not only to obtain information related to the patient's health in the clinic and to evaluate the functional problems of the patients, but also it is used

Received Jun 9, 2021 Revised Jun 16, 2021

Accepted Jun 22, 2021

Corresponding author Jung-Won Kwon

E-mail kjwonpt@hanmail.net in various fields as an effective tool to develop appropriate intervention strategies in the process of clinical reasoning., ${ }^{2,3}$

Multiple ligament injury of the knee joint refers to a case where two or more ligaments are damaged, it is also used mixing with knee dislocation, a condition in which tibio-femoral articulation is impaired. ${ }^{4}$

Dislocation of the knee joint is mainly caused by high energy impact such as pedestrian accident due to car accident, ${ }^{5,6}$ and, in long-term point view, cause serious functional limitations. ${ }^{7}$ If proper treatment is not performed, it affects the treatment results, instability and arthritis occur, a therapeutic approach based on accurate anatomical understanding of the knee joint is needed. ${ }^{8,9}$

The knee joint maintains static stability by dynamic stability by the 
muscles around the joint, anterior cruciate ligament, posterior cruciate ligament, and medial and lateral collateral ligament, meniscus, which maintains the gap between tibia and the femur, also contributes to the stability of the knee joint. ${ }^{8}$ Among these structures, ligament damage is a very important structure for knee joints because it significantly lowers knee joint stability and causes traumatic arthritis, causing pain and limited movement. ${ }^{4,10}$

MRI (Magnetic Resonance Imaging) is the most accurate method for examining and evaluating knee dislocation, upon dislocation of the knee joint, $100 \%$ of the anterior cruciate ligament, $88 \%$ of the posterior cruciate ligament, 35\% of the collateral ligament and $46 \%$ of the hamstring tendon were reported. ${ }^{11}$ For this treatment requires a comprehensive and appropriate evaluation of ligament damage as well as nerve vessels for a positive prognosis. $^{12,13}$

Zhang et al. ${ }^{14}$ reported that neurovascular and structural damage to the knee joint has a serious impact on the biomechanical changes in the human body. Also, maintaining the correct body alignment has the advantage that the minimum consumption of muscle energy requirement, ${ }^{15} \mathrm{es}-$ pecially it is emphasized that the correct sitting position is important because the correct sitting position is closely related to the muscle energy consumption in the standing position by maintaining correct body alignment. ${ }^{16}$

The sitting cross-legged posture is a posture commonly found in Asian cultures where sedentary lifestyle is commonly, and the mechanical form shows the form of flexion, abduction and external rotation of the hip joint, ankle joints show plantar flexion and dorsi-flexion, and inversion and eversion in a rather little range. ${ }^{1718}$ For the average angle of the sitting cross-legged posture, Kapoor et al..$^{17}$ reported $91^{\circ}$ flexion, $39^{\circ}$ abduction and external rotation $49^{\circ}$ for the hip joint, the knee joint was flexion at $135^{\circ}$, and the ankle joint dorsiflexion was $23^{\circ}$. Also, Zhou et al.$^{18}$ found that the hip flexion in the sagittal plane was $101.7^{\circ}$, the knee joint flexion was $131.9^{\circ}$, the ankle joint dorsi-flexion was $12.3^{\circ}$, and the plantar flexion was $20.2^{\circ}$. In the movement of the frontal plane, the average abduction of the hip joint was $43.2^{\circ}$, inversion of the ankle joint was $22.8^{\circ}$, eversion was $12.3^{\circ}$, the external rotation of the hip joint was $36.4^{\circ}$, and the internal rotation of the knee joint was $32.4^{\circ}$. Therefore, the sitting cross-legged posture requires high level movement from the hip joint to the ankle joint. The most important purpose to be considered in the rehabilitation process is to restore joint motion and function and muscle strength in a pain free range to return to daily life. There are not many cases of sitting crosslegged posture for multiple ligament damage, and have many variables.
Therapists need to develop the most effective intervention strategies with patients for accurate treatment, and in order to do. They need to gather a lot of information from patients, analyze and identify problems, and support the therapist's accurate decision-making.

The purpose of this case study is to provide an appropriate intervention strategy for the sitting cross-legged posture, a high level of movement, to return to a smooth society of patients with multiple ligament injuries of the knee joint by applying the ICF checklists.

\section{PATIENT INFORMATION}

\section{Patient information}

In this case study, 29-year-old unmarried man who served as a teacher. He was $178 \mathrm{~cm}$ height and $68 \mathrm{~kg}$ weight.

\section{Diagnosis and history}

After a car accident on the highway, he had undergone skin grafts on the lower right leg, the outer tibia, and the back side and fracture of the left tibia and dislocation of the right knee joint were diagnosed with multiple ligament injuries of the right knee, tibia nerve injury, and stiffness of the right knee. Before a car accident, he had been no special treatment or surgery.

\section{Environment and social information}

He was a high school teacher based in J city and was in graduate school. He enjoyed soccer and baseball in a free time, and had frequent meetings with friends, so he drank alcohol once every two days and did not smoke. He lived alone in a hospital without a caregiver.

\section{Patients need and attitude}

After returning from work, the patient asked for I would like to sit down and eat in a sitting cross-legged posture at the dinner table, and willingness to rehabilitate was strong, but he did not like to do difficult exercises. Also, complained of depression due to changes in skin color and scarring and anxiety about knee condition due to skin grafts.

\section{Patient's functional activity level and symptoms}

As a result of the posture change test, "rolling", "laying sides", and "proneness" were possible to determine the patient's functional activity level, but when changing from a standing position to a sitting cross-legged posture, he complained of difficulties. Also, it was possible to "sit to stand", but dif- 
ficulty in squatting and maintaining sitting cross-legged posture complained. Walking was only possible using crutch. As a result of confirming the general sensation, he was a decrease in superficial sensation such as thalposis, pressure sense, and cooling of the lower extremities of both legs, and no light touch feeling. In the Integumentary system, there was a decrease in elasticity and changes in skin color due to skin graft after a car accident.

\section{CLINICAL FINDINGS}

\section{Range of Motion and Length}

To measure the range and range of motion of the patient by using a goniometer and tape measure. As a result of examining the range of motion of the joints in sitting cross-legged posture, the range of motion of the knee joint was $110^{\circ}$, and the knee joint flexion length (ASIS- medial condyle of tibia) was $69 \mathrm{~cm}$.

\section{Manual Muscle Test (MMT)}

To evaluate the patient's muscular strength, a manual muscle test was performed. As a result of the strength test, the left lower extremity muscle was normal, the right lower extremity knee flexion was Poor-, and the extensor was Poor, the right ankle joint dorsi-flexor was Poor and the plantar flexor Poor-showed weakness.

\section{Instability of the knee joint}

For the knee joint instability test, based on the study by Anderson et al. ${ }^{19}$ anterior drawer test for the anterior cruciate ligament rupture and a Lachman's test were performed, a reverse deflection test and reverse Lachman's test were performed for the posterior cruciate ligament rupture. The medial and lateral stress tests were performed for the medial collateral and lateral collateral ligament rupture tests. Dial test was performed as a posterior structural complex test. As a result of the test, the anterior drawer test was less than $6 \mathrm{~mm}$, and the Lachman's test showed that the tibia forward on the femur and the end feel when the knee bone tendon slope disappeared was all negative (-). The posterior draw test was negative (-), because there was no fluctuation compared to the left lower extremity, even in the shaking of less than $6 \mathrm{~mm}$ and in the reverse Lachman's test. The medial and lateral ligament stress tests were negative (-) because the tibia did not move from the femur. The result of the dial test was negative (-) when there was no significant difference when comparing the tibia and rotational force of the left lower extremity.

\section{Dynamic balance}

To evaluate the patient's dynamic balance ability Berg Balance Scale (BBS) was performed.$^{20} \mathrm{BBS}$ is divided into three categories: "sitting," "standing", and "posture change", with a total of 14 items, applying a minimum of 0 to a maximum of 4 points for each item, and a total of 14 items for 56 points, less than 20 points means "high risk of falling", 21-40 points means "moderate fall risk", and 41-56 points means "low risk of falling" ${ }^{20}$ That results, the patient had a low risk of falling to 52 points.

\section{Pain}

Visual analogue scale (VAS) was used to investigate knee joint pain when maintaining sitting cross-legged posture and ordinary. ${ }^{21} 0$ points was no pain, 5 points was moderate pain, 10 points was uncontrollable pain, and the patient's pain level was 3 points on average and 7 points on maintaining sitting cross-legged posture.

\section{Depression}

Korea version Beck Depression Inventory (BDI), which is widely used as a self-report scale to determine the patient's current psychology was used.22 BDI consists of 21 questions including the cognitive, emotional, motivational, and physical symptoms areas of depression, and the higher the total score from 0 points to 3 points for each question, the higher the total score, the greater the degree of depression. The amputation point of BDI is classified as 16-19 points depression aspect, 20-23 points depression, and 24 points or higher as severe depression. ${ }^{23}$ As a result of examining the patient's depression level, he had a severe depression with 41 points.

\section{Sitting cross-legged}

A stopwatch was used to test the ability to maintain the sitting crosslegged posture, and the maintenance was 30 seconds.

\section{DIAGNOSTIC ASSESSMENT}

\section{ICF checklists}

As an evaluation index that provides a comprehensive synopsis of the individual's function on the current evaluation and results in human function, the upper part (body function, structure, activities and participation) shows the patient's perspective and the lower part (personal and environmental factors) shows the health expert's perspective (Figure 1). ${ }^{24}$

The patient complained of difficulty in a squatting, maintaining a squatting posture, and maintaining a sitting cross-legged posture. The 


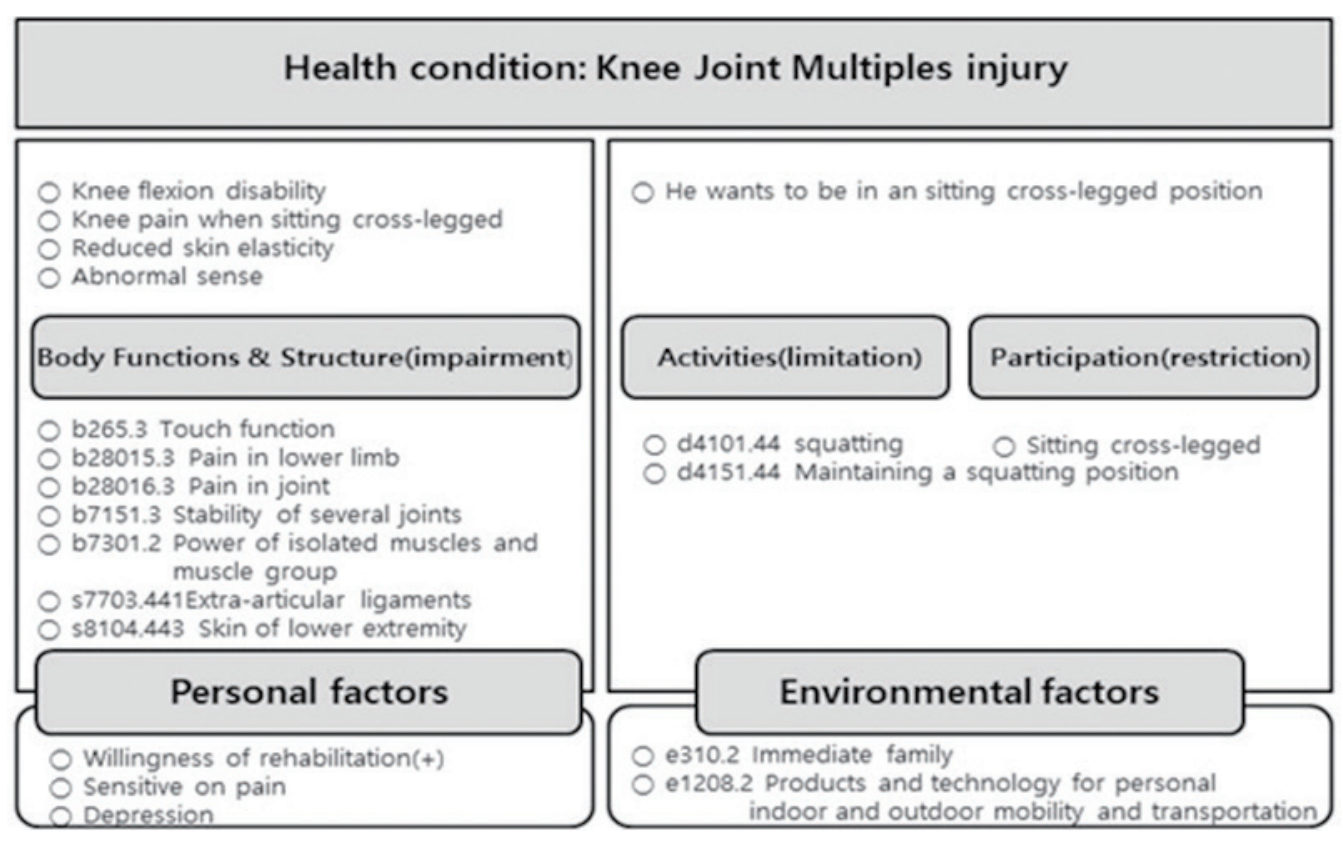

Figure 1. ICF Assessment Sheet.

problem of the level of damage to this was "the knee is not flexed", "the knee pain in sitting cross-legged postures", "there is a decrease in elasticity due to both legs skin grafts", "there is damage to the touch".

At the level of activity and participation, Noticing the problem of "difficulties maintain sitting cross-legged posture in the restaurant".

\section{1) Physical and structural damage}

In the area of 'body function and structural damage', the strength of one limb muscle's severely damage (25-49\%), mobility of multiple joints, pain in the lower limbs, pain in the joints, and touch function's highly damage (50-95\%), Ligaments of the skin and joints of the lower limbs complete damage (96-100\%) were able to confirm.

\section{2) Restriction of activity and participation}

In the area of 'Activity and Participation', the restriction of activities that do not maintain the posture of sitting cross-legged in the restaurant is related to the restriction of participation in 'returning to work and performing eat', the above activity showed high (50-95\%) damage.

\section{3) Environment and personal factors}

The "personal factor" was depression due to pain and did motivate, but he did not like to do difficult exercises. "Environmental factor", was living alone without a caregiver and being too dependent on crutches served as a moderate barrier.

\section{4) ICF interaction between each item}

As a result of coding ICF checklists obtained factual information through therapist's observational analysis and interview of the patient's required activity limitations and participation constraints and problems and found that the following interactions exist. Patient had restrictions on activities such as "maintaining sitting cross-legged posture" regarding participation restrictions such as eating in a restaurant.

This is "b7301.2 Power of isolated muscles and muscle group" in physical function, "b7151.3 Stability of several joint", 'b28015.3 Pain in lower limb', 'b28016.3 Pain in joint', 'b265.3 Touch function', and 's8104.443 Skin of lower extremity', s7703.441 Extra-articular ligaments was found to be a factor influencing 'd4101.44 Squatting', and d4151.44 Maintaining a squatting position' and the maintenance of sitting cross-legged posture. In addition, in terms of environmental factors, the rehabilitation will be strong as a personal factor, but it showed a very sensitive response to pain and scars on the skin of the lower limbs, and depressed by these factors, complaining of dullness of touch function.

\section{Decision-making process}

\section{1) Main activity limits}

Maintaining sitting cross-legged posture.

\section{2) Hypothesis}

Hypotheses were prepared for the reasons why it is difficult to change and 
maintain the sitting cross-legged posture.

(1) Does the limitation of the lower limbs range cause limitations in sitting cross-legged posture?

It was judged that the angle of the knee flexion was notably low due to the knee joint flexion range of $110^{\circ}$, so that sitting cross-legged posture did not appear.

(2) Does muscle weakness cause limitations in knee flexion?

According to Kisner, ${ }^{15}$ when the knee flexion, the hamstring muscle is the primary flexor that affects the tibia rotation. In addition, the knee extensor controls the degree of flexion, and the ankle joint plantar flexor is said to play a main function in knee flexion in the open chain.

As a result of manual muscle test, the right lower extremity muscle strength, a significant decrease in knee flexor was evaluated. Based on this fact, it was judged that the normal flexion angle did not come out due to the lack of muscle strength needed to flexion the knee.

(3) Does knee joint instability cause sitting cross-legged posture limitations? To examine the knee instability of the patient, for anterior cruciate ligament rupture, anterior drawer test, and Lachman's test were performed, and for the posterior cruciate ligament rupture, posterior tibia draw test and a reverse Lachman's test were performed. In addition, Dial test was performed by examining the medial and lateral for medial ligament and lateral ligament rupture, and the posterior lateral complex. All of the evaluation results were negative (-), so it was judged that there was no knee joint instability in the patient.

(4) Does the dynamic balance ability of the knee joint cause limitations in sitting cross-legged posture?

Once, evaluating the patient's movement, the BBS test was performed to determine that dynamic balance ability had an effectiveness on posture change, when both feet were opened to widen the support surface when sitting. However, as a result of the measurement, it scored 52 points out of a total of 56 points, indicating that there was a low risk of falling, indicating that the dynamic balance ability did not decrease.

\section{(5) Does pain cause sitting cross-legged posture limitations?}

Patient complains of persistent knee pain, it was judged to affect the maintenance of sitting cross-legged posture. VAS was used to evaluate the degree of pain, and as a result of the evaluation, it was found that the pain was less than moderate with 3 points in normal, and the pain was more than moderate with 7 points in sitting cross-legged posture. As a result, it was judged that the pain affects sitting cross-legged posture.

\section{(6) Does depression cause sitting cross-legged posture limitations?}

The rehabilitation willing was strong, but was sensitive to pain and scars on lower extremity skin graft, complaining of touch dullness. He often said that he was depressed by these causes, to confirm this, the BDI test was conducted, and it was a serious condition with 41 points. Therefore, the depression has a negative effect on rehabilitation, it was judged that postured sitting cross-legged posture.

\section{3) List of problem}

(1) Limit of the range of motion of the right lower limb joint

(2) Weakening of the knee flexor

(3) Knee pain

(4) Depression

\section{Diagnosis}

After discharge, the patient wanted to eat with sitting cross-legged posture, and based on the resolution tasks negotiated with the patient, the most important function limit was 'Maintaining sitting cross-legged posture'. The problems affecting this were judged to be due to the limited range of motion, weakened muscle strength, knee pain, and depression.

\section{Prognosis}

In order to maintain the targeted posture of sitting cross-legged, recovery of ROM in the lower right limbs, muscle strength recovery, pain control, and recovery of depression are thought to have a positive effect on 'Maintaining the posture of sitting cross-legged'.

(1) Short term goal: After 4 weeks, can squat for sitting cross-legged.

(2) Long term goal: After 6 weeks, can eat while maintaining sitting cross-legged posture.

\section{THERAPEUTIC INTERVENTION}

The patient had a limitation in the range of motion of the right lower extremity and complained of muscle weakness, pain, declining ability to maintain sitting cross-legged posture, and depression. For the intervention, progressive strength training and range of motion training were per- 
formed 30 times a day, 5 times a week for 6 weeks.

\section{Range of motion exercise}

1) Gliding exercise of the knee in the supine position (Figure 2A).

2) Posterior gliding of the tibia in the supine position (Figure 2B).

3) In a standing position, put feet on the board and self-extension stretching (Figure 2C).

4) Hold and relax technique to increase knee flexion in the supine position (Figure 2D).

\section{Muscle strength}

1) Knee flexion exercise in standing position (Figure 3A).

2) Heel raise exercise with $1 / 3$ of the sole on the board in standing position (Figure 3B).

3) Knee flexion exercise after internal rotation of the tibia in supine position (Figure 3C).

4) Plantar flexion and dorsi-flexion exercise in bridge position (Figure 3D).

5) Knee extension exercise with sandbag in sitting position (Figure 3E).

6) Knee extension exercise with manual resist in sitting position (Figure $3 \mathrm{~F})$.
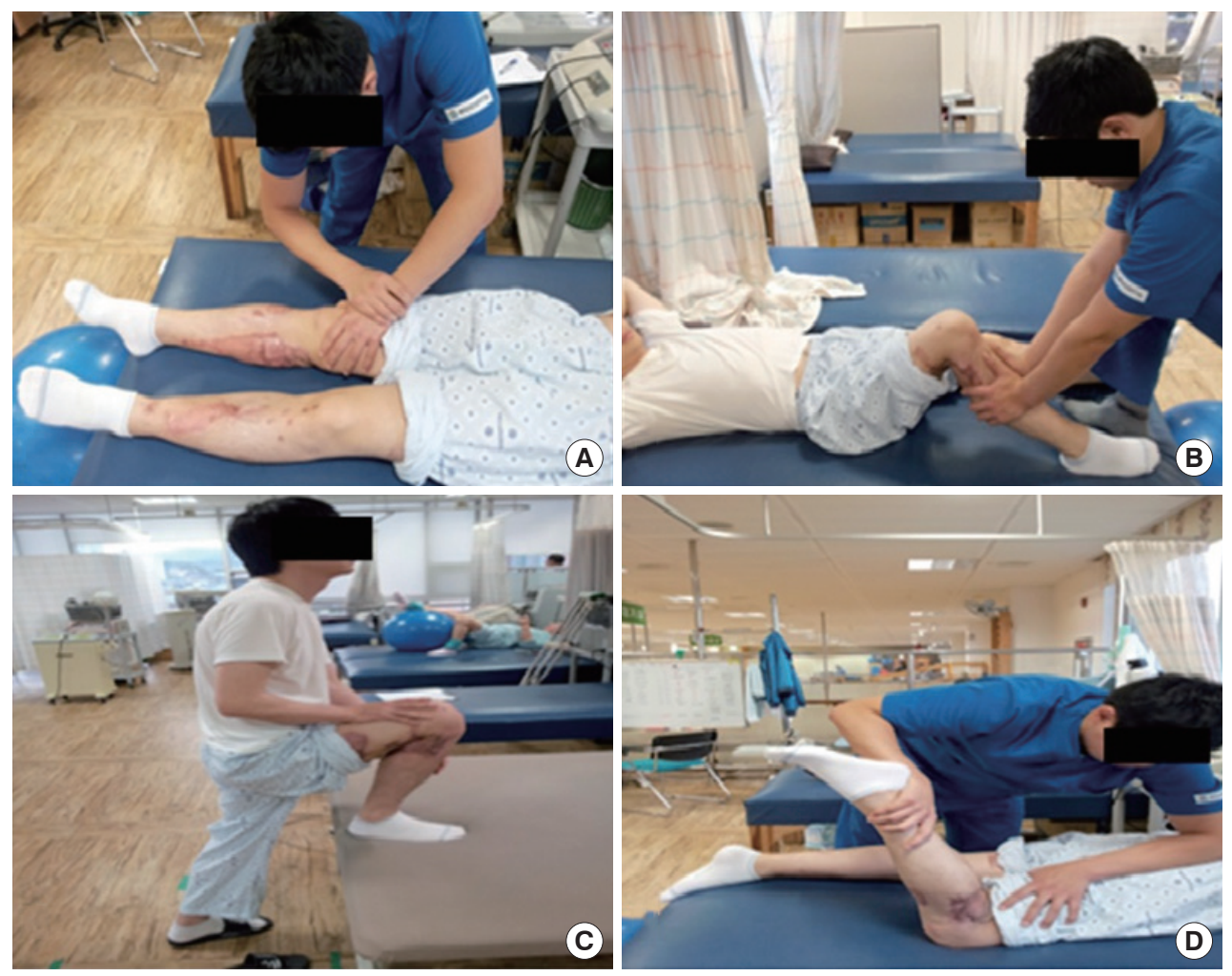

Figure 2. Range of motion exercise. (A) Inferior gliding of patella, (B) Posterior gliding of tibia, (C) Self-stretch with stimulus, (D) Hold \& relax for knee flexion.

\section{Pain control}

1) Hot pack

2) Ultrasound

3) transcutaneous electrical nerve stimulation

\section{Depression control}

In order to control patient depression, counsel patient in the treatment room, recommend balanced eating, reading, and walking. Also, beware of alcohol or illegal drugs.

\section{OUTCOMES}

\section{Range of motion and Length}

The range of motion of the knee joint was improved from $110^{\circ}$ before intervention to $135^{\circ}$ after intervention, and the knee joint flexion length was reduced from $69 \mathrm{~cm}$ to $45 \mathrm{~cm}$ (Table 1 ).

\section{Manual Muscle Test}

The knee flexors improved from Poor- before intervention to Good after intervention, extensor muscle improved from Poor before intervention to 

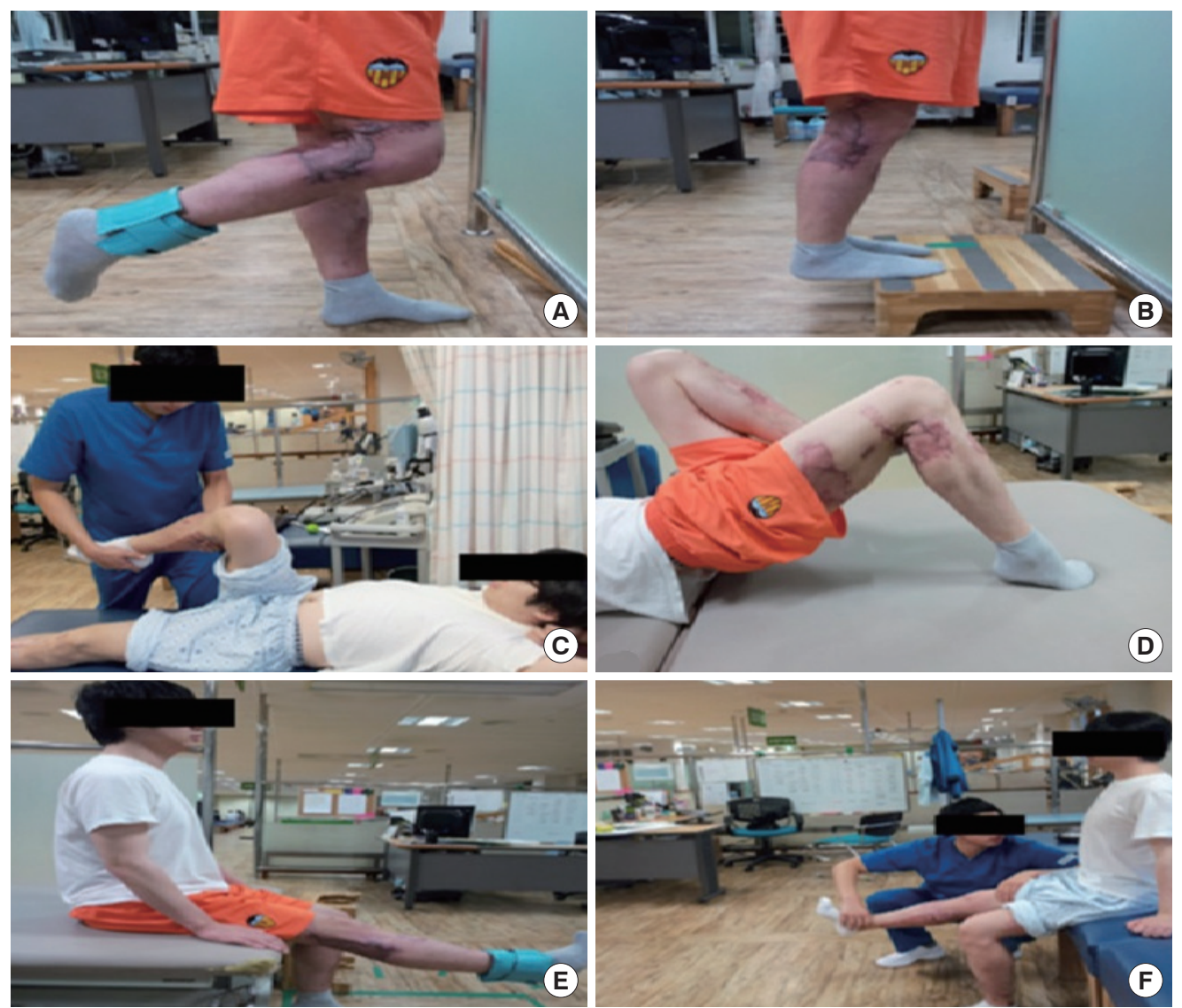

Figure 3. Muscle strengthening exercise. (A) Knee flexion exercise with sandbag, (B) Heel raise exercise, (C) Knee flexion in tibia internal rotation, (D) Plantar flexion \& dorsi flexion on bridge position, (E) Knee extension with sandbag, (F) Knee extension with resistance by therapist.

Table 1. Outcomes

\begin{tabular}{lcc}
\hline Item & Pre-intervention & Post-intervention \\
\hline Knee joint Range of Motion & $110^{\circ}$ & $135^{\circ}$ \\
Length of Knee joint flexion & $69 \mathrm{~cm}$ & $45 \mathrm{~cm}$ \\
Knee flexor strength & Poor- & Good \\
Knee extensor strength & Poor & Good+ \\
Ankle plantar-flexor strength & Poor- & Good \\
Ankle dorsi-flexor strength & Poor & Good \\
Visual analogue scale & & \\
$\quad$ Daily & $3 \mathrm{score}$ & $2 \mathrm{score}$ \\
$\quad$ Sitting cross-legged & $7 \mathrm{score}$ & 4 score \\
Depression score & $41 \mathrm{score}$ & $31 \mathrm{score}$ \\
Sitting cress-logged time & $30 \mathrm{sec}$ & $14 \mathrm{~min}$ \\
\hline
\end{tabular}

Good+ after intervention, the plantar flexor of the ankle joint improved from Poor- before intervention to Good after intervention, the dorsi flexor muscle improved from Poor before intervention to Good after intervention (Table 1).

\section{Pain}

The patient's pain level was 3 points before the intervention and 2 points after the intervention, when maintaining the sitting cross-legged posture, the score before intervention was 7 to 4 after intervention, indicating a moderate or lower pain index (Table 1).

\section{Depression}

Beck Depression Inventory decreased by 10 points from 41 points before intervention to 31 points after intervention, but the results did not change to severe depression (Table 1).

\section{Maintain the sitting cross-legged posture}

As a result of measuring the maintaining sitting cross-legged posture, it increased by about 13 minutes and 30 seconds from 30 seconds before intervention to 14 minutes after intervention (Table 1).

\section{The changes of ICF checklists}

As a result of functional level evaluation using ICF checklists (Table 2), the 
Table 2. The changes of ICF checklists

\begin{tabular}{lcc}
\hline ICF checklists & Pre-intervention & Post-intervention \\
\hline Stability of a several joint & $\mathrm{b} 7151.3$ & $\mathrm{~b} 7151.1$ \\
Power of muscles of one limb & $\mathrm{b} 7301.2$ & $\mathrm{~b} 7301.1$ \\
Pain in lower limb & $\mathrm{b} 28015.3$ & $\mathrm{~b} 28015.1$ \\
Pain in joint & $\mathrm{b} 28016.3$ & $\mathrm{~b} 28016.1$ \\
Squatting & $\mathrm{d} 4101.44$ & $\mathrm{~d} 4101.11$ \\
Maintaining a squatting position & $\mathrm{d} 4151.44$ & $\mathrm{~d} 4151.11$ \\
Skin of lower extremity & $\mathrm{s} 8104.44$ & $\mathrm{~s} 8104.44$ \\
Touch function & $\mathrm{b} 265.3$ & $\mathrm{~b} 265.3$ \\
\hline
\end{tabular}

ICF, International Classification of Functioning.

patient showed high level of Stability of several joints (b7151.3) as the Stability of several joints with mild (b7150.1), and power of isolated muscles and muscle group (b7301.2) power of isolated muscles and muscle group (b7301.1), the high level damage of the pain in lower limb (b28015.3) is the mild pain in lower limb (b28015.1), and the high level of the pain in joint (b28016.3) were reduced to mild pain in joint (b28016.3).

Squatting (d4101.44) of extreme severity (96-100\%) damage is Squatting (d4101.11) of mild, the extreme severity of maintaining a squatting position and sitting cross-legged posture (d4151.44) were reduced to the level of maintaining a squatting position and sitting cross-legged posture (d4151.11). there was no change in skin of lower extremity (s8104.44) and touch function (b265.3).

\section{DISCUSSION}

Multiple ligament injuries of the knee joint are fatal to the knee joint because of soft tissue and neurovascular damage, depending on the amount of impact and the mechanism of injury. ${ }^{25,26}$ Rehabilitation of ligament injury is an important factor in the proper balance of range of motion and functional recovery, ${ }^{27}$ and muscle strengthening also contributes to the reduction of pain and edema during the rehabilitation process. ${ }^{27,28}$ According to a precedent study, Reynold ${ }^{29}$ reported that the range of motion, pain, and muscle weakness have functional problems and interaction effects, so a complex treatment approach with anatomy and pathology is needed and treatment should be applied in a variety of tools and conditions rather than applying a uniform program in general principles. Mook et $\mathrm{al}^{30}$ reported that range of motion and strength training have a positive effect on knee stability in patients with multiple ligament injury, it is thought that progressive range of motion, strength training and pain control will have a positive effect on the improvement of knee joint function.

The results of this study also showed that the knee flexion range of pa- tients improved from $110^{\circ}$ before intervention to $135^{\circ}$ after intervention, and the knee flexion length decreased from $69 \mathrm{~cm}$ to $45 \mathrm{~cm}$. Also, in the muscle strength, the knee flexor is Good after intervention from Poor- before intervention, extensor improved from Poor before intervention to Good+ after intervention, the plantar flexor of the ankle joint is also Good after intervention in Poor-, the dorsi flexor improved from Poor before intervention to Good after intervention.

In the ICF function level changes, the high level of stability of several joint (b7151.3) is stability of several joints with a mild (b7151.1), the power of isolated muscles and muscle group (b7301.2) was positive with the power of isolated muscles and muscle group (b7301.1) of mild. These results demonstrate that Kisner and Colby ${ }^{15}$ wrote that knee bone synovial membrane, knee joint back synovial membrane and self-extension improve the range of motion of the knee joint, and demonstrate Milsom et al. reports that gradual resistance exercise increases muscle strength.

The change of visual analogue scale decreased from 3 points in the usual visual analogue scale to 2 points after intervention, when maintaining the sitting cross-legged posture, it showed a positive effect in reducing the visual analogue scale below moderate, from 7 points before intervention to 4 points after intervention.

Changes in ICF ranged from severe pain in lower limb (b28015.3) to mild pain in lower limb (b28015.1), high pain in joint (b28016.3) was reduced to mild pain in joint (b28016.3). It indicates that strengthening the hamstring muscle has a positive effect on pain reduction, ${ }^{32} \mathrm{Kim}$ and $\mathrm{Ahn}^{33}$ said that when applying transcutaneous nerve stimulation, it is effective in controlling pain by activating coarse nerve fibers in the vicinity of pathologically damaged nerve fibers. In addition, it has been reported that ultrasound therapy has a positive effect on pain reduction by raising deep heat, ${ }^{34}$ it is thought that the strength exercise applied in this study and general physical therapy were effective.

In this study, the patient's requirements and the final goal, 'sitting crosslegged' posture increased from 13 seconds to 14 minutes before intervention as a result of measurement of retaining time. In addition, squatting (d4101.44) with severe damage is squatting (d4101.11) with mild damage, the severe of maintaining a squatting position and sitting cross-legged posture (d4151.44) were reduced to the level of mild damage maintaining a squatting position and sitting cross-legged posture (d4151.11). As shown in the above results, it seems that the improvement of joint motion range and knee length, increased muscle strength, and reduced pain affected the positive effects.

Although the fact that it is a single subject study has a disadvantage of 
limiting the generalizability of a study's results, the ultimate goal of rehabilitation is to improve the quality of life by acquiring sufficient information from patients and bringing functional improvement to the full potential of people with disabilities. So, this study, apply the ICF checklists, patients with multiple ligament injuries were identified by dividing the patient's problems into body functions and structural areas representing changes in the body's physiological functions and anatomical structures, it is meaningful that the patient has fulfilled the requirements for daily living rehabilitation by grasping complexly the activities and participation areas, including the ability to perform certain tasks. Also, after understanding the environmental factors and individual factors in detail, design hypothesis and approach. Patient-centered treatment is very important. By using ICF, it was possible to establish a common goal of maintaining the "sitting cross-legged posture" between the therapist and the patient, because of this approach, it was possible to induce successful rehabilitation and spontaneous participation of patients.

\section{REFERENCES}

1. World health organization. World health organization staff. international classification of functioning, disability and health. World Health Organization. 2001.

2. Lee MJ, Kim H. Factors related to job performance of female patients with workplace injuries by using ICF model. J Korean Soc Phys Med. 2018;13(2):21-31

3. Yun YN, Choi GE, Lim HW. Assessment using the ICF-core set for children and youth with cerebral palsy: a single case study. J Kor Phys Ther. 2019;31(2):103-10.

4. Kim WM, Chun CC. The management of knee dislocation and multiple ligament injuries. J Kor Sports Med. 2012;30(1):1-8.

5. Chhabra A, Klimkiewicz JJ, Petrie RS et al. The dislocated knee Insall and Scott surgery of the knee. 4th ed. Philadelphia, Churchill Livingston, 2011:756-75.

6. Shelbourne KD, Haro MS, Gray T. Knee dislocation with lateral side injury: results of and en masse surgical repair technique of the lateral side. Am J Sports Med. 2007;35(7):1105-16

7. Maxwell GT, Warth RJ, Amin A et al. Multiple ligament knee injured: does the knee dislocation classification predict the type of surgical management? J Knee Surg. 2021;34(3):273-9.

8. Choi CJ, Choi CH, Oh WT. Treatment of multiple ligaments injuries. J Korean Knee Soc. 2010;22(2):82-92.

9. Fanelli GC. The multiple ligament injured knee: a practical guide to management. 2nd ed. New York, Springer. 2019:242.

10. Almekinders LC, Logan TC. Results following treatment of traumatic dislocations of the knee joint. Clin Orthop Relat Res. 1992;284:203-7.

11. Kannus P, Jarvinen M. Nonoperative treatment of acute knee ligament injuries. Sports Med. 1990;9(4):244-60.
12. Miranda FE, Dennis JW, Veldenz HC et al. Confirmation of the safety and accuracy of physical examination in the evaluation of knee dislocation for injury of the popliteal artery: a prospective study. J Trauma Acute Care Surg. 2002;52(2):247-52.

13. Helgeson MD, Lehman RA, Murphy KP. Initial evaluation of the acute and chronic multiple ligament injured knee. J Knee Surg. 2005;18(3): 213-9.

14. Zhang L, Liu G, Han B. Knee joint biomechanics in physiological conditions and how pathologies can affect it: a systematic review. Appl Bionics Biomech. 2020;2020:7451683.

15. Kisner C, Colby LA. Therapeutic exercise: foundations and techniques. Philadelphia, FA Davis, 2020:265.

16. Kendall DF, McCreary EK, Provance PG. Muscles, testing and function: with posture and pain. Baltimore, Williams \& Wilkins. 1993:103.

17. Kapoor A, Mishra SK, Dewangan et al. Range of Movements of lower limb joints in cross-legged sitting posture. J Arthroplasty. 2008;23(3): 451-3.

18. Zhou H, Liu A, Wang D et al. Kinematics of lower limbs of healthy Chinese people sitting cross-legged. Prosthet Orthot Int. 2013;37(5):369-74.

19. Anderson MK, Hall SJ, Martin M. Foundations of athletic training: prevention, assessment, and management. Philadelphia, Lippincott Williams \& Wilkins. 2004:620.

20. Berg KO, Wood-Dauphinee SL, Williams JI et al. Measuring balance in the elderly: validation of an instrument. Can J Public Health. 1992;83:711.

21. Wewers ME, Lowe NK. Acritical review of visual analogue scales in the measurement of clinical phenomena. Res Nurs Health. 1990;13(4):22736.

22. Hahn H. A standardization study of beck depression inventory in Korea. J Korean Neuropsychiatr Asso. 1982;25:487-502.

23. Rhee M. A Standardization study of beck depression inventory I-Korean version (K-BDI): reliability and factor analysis. Korean J Psychopathol. 1995;4:77-95.

24. Rauch A, Cieza A, Stucki G. How to apply the international classification of functioning disability and health (ICF) for rehabilitation management in clinical practice. Eur J Phys Rehabil Med. 2008;44(3):329-42.

25. Moatshe G, Chahla J, LaPrade RF et al. Diagnosis and treatment of multiligament knee injury: state of the art. J ISAKOS: Jt Disord Orthop Sport Med. 2017;2(3):152-61.

26. Wascher DC, Dvirnak PC, DeCoster TA. Knee dislocation: initial assessment and implications for treatment. J Orthop Trauma. 1997;11(7): 525-9.

27. Edson CJ, Fanelli GC, Beck JD. Rehabilitation after multiple-ligament reconstruction of the knee. Sports Med Arthrosc. 2011;19(2):162-6.

28. Palmieri-Smith RM, Thomas AC, Wojtys EN. Maximizing quadriceps strength after ACL reconstruction. Clin Sports Med. 2008;27(3):405-24.

29. Reynolds N. Functional rehabilitation of sports and musculoskeletal injuries. J Athl Train. 1999;34(1):70.

30. Mook WR, Miller MD, Diduch DR et al. Multiple-ligament knee injuries: a systematic review of the timing of operative intervention and postoperative rehabilitation. JBJS. 2009;91(12):2946-57.

31. Milsom J, Barreira P, Burgess DJ et al. Case study: muscle atrophy and hypertrophy in a premier league soccer player during rehabilitation from ACL injury. Int J Sport Nutr Exerc Metab. 2014;24(5):543-52. 
32. Bokaeian HR, Bakhtiary AH, Mirmohammadkhani M et al. Quadriceps strengthening exercises may not change pain and function in knee osteoarthritis. J Bodyw Mov Ther. 2018;22(2):528-33.

33. Kim JM, Ahn DH. Pain physiology and principles of physical therapy.
Phys Ther Korea. 1998;5(2):106-17.

34. Griffin JE. Physiological effect of ultrasonic energy as it is used clinically. Phys Ther. 1966;46(1):18-26. 